\title{
The State and Future Directions of Atom Probe Tomography
}

\author{
M.K. Miller* \\ * Metals and Ceramics Division, Oak Ridge National Laboratory, PO Box 2008, Oak Ridge, TN \\ 37831-6136
}

Fifty years ago, Müller and Bahadur performed field ion microscopy experiments in which images of individual atoms were obtained for the first time. Over a decade later, Müller, Panitz and McLane added a time-of-flight mass spectrometer to that instrument so that the individual atoms could be identified [1]. This atom probe field ion microscope featured a hemispherical glass ball joint so that the apex of the specimen could be rotated to align the atom of interest with a small hole in the phosphor screen on which the field ion image was formed. This aperture served as the entrance aperture to the time-of-flight mass spectrometer. The flight time and hence the mass-to-charge ratio of each selected atom field evaporated from the specimen was measured with an oscilloscope. Many technological advances have improved the design. These advances include helium cryogenerators to cool the specimen to cryogenic temperature, solid state pulse generators to field evaporate the atoms from the specimen, computer controlled digital timing systems to measure the flight times, and position-sensitive single atom detectors to enable the coordinates of the atoms to be determined [2].

A state-of-the-art local electrode atom probe is shown schematically in Fig. 1. In order to accurately align the cryogenically-cooled specimen with the 10-50 $\mu \mathrm{m}$ diameter aperture in the funnel-shaped electrode, the interchangeable specimen is mounted on a nanopositioning XYZ stage. A high speed $(\leq 250 \mathrm{kHz})$ solid state pulse generator applies negative voltage pulses to the local electrode to field evaporate ions from the positively biased specimen. The flight times and the $\mathrm{X}$ and $\mathrm{Y}$ position of the impact of the ions are measured with a crossed delay line position-sensitive detector coupled to a high speed computer-controlled digital timing system. Because energy-compensating lenses are not necessary, the detector may be positioned close to the specimen resulting in a wide field of view, typically 50-100 $\mathrm{nm}$. These improvements result in substantially more atoms being collected from the specimen, typically 250,000 to 100 million atoms. Data collection rates of up to 2 million atoms per minute have been achieved. Under development are instruments that use pulsed lasers to assist in the field evaporation process and should enable higher resistivity materials to be analyzed. An example of an analysis is shown in Fig. 2.

Traditionally, most atom probe needle-shaped specimens have been fabricated by electropolishing methods. Dual beam focused ion beam (FIB) instruments have enabled suitable specimens to be fabricated from a significantly wider range of materials. Because of the high resolution capabilities of the FIB, specimens can also be cut from site specific regions [3]. Specimens can also be fabricated from films or multilayers deposited on substrates, as shown in Fig. 3 for a metallic layer deposited on a post that was Bosch etched from a silicon wafer. Other methods have also been used to produce arrays of sharp needles on which films may be deposited, Fig. 4. By moving the specimen, an individual needle or post may be aligned with the local electrode for analysis [4].

[1] E. W. Müller, J. A. Panitz and S. B. McLane, Rev. Sci. Instrum., 39 (1968) 83.

[2] M.K. Miller, Atom Probe Tomography, Kluwer Academic/Plenum Press, New York, 2000.

[3] M.K. Miller, K.F. Russell and G.B. Thompson, Ultramicroscopy, 102 ( 2005) 287. 
[4] Research at the SHaRE User Facility was sponsored by the Division of Materials Sciences and Engineering, U. S. Department of Energy, under Contract DE-AC05-00OR22725 with UT-Battelle, LLC.

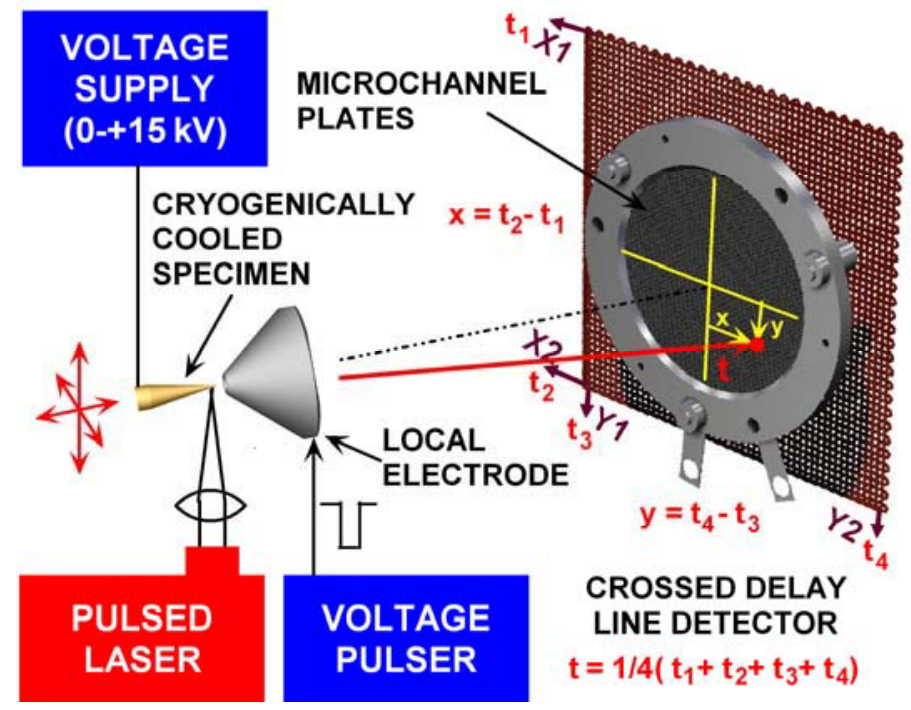

FIG. 1. Schematic diagram of a local electrode atom probe. Two alternative methods of field evaporation ions from the specimen are shown -voltage and laserassisted pulsing.

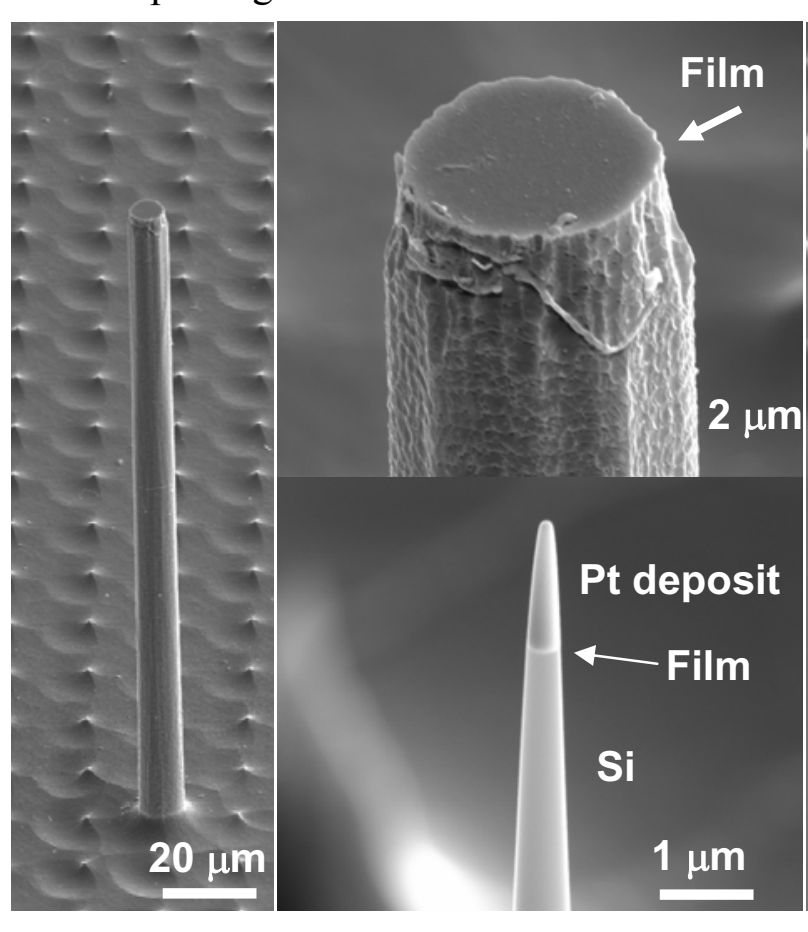

FIG. 3. A partially finished atom probe specimen being fabricated with the use of a FIB from a metallic film deposited on a post that was Bosch etched from a Si wafer.

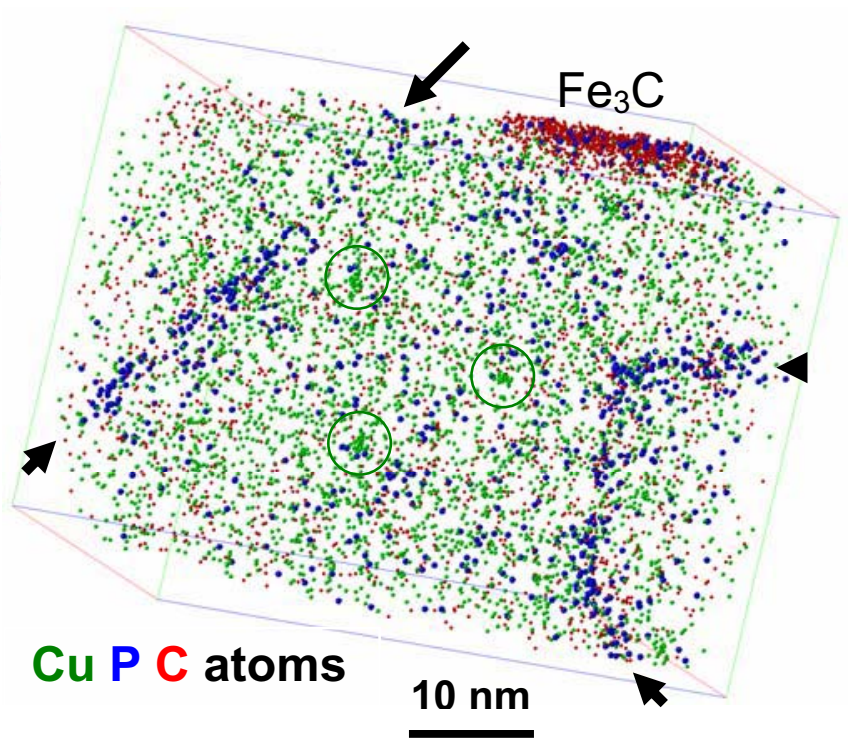

FIG. 2. Atom map of two P-enriched dislocations (arrowed), $\mathrm{Fe}_{3} \mathrm{C}$ and 2-nmdiameter copper-enriched precipitates (circled) in a neutron-irradiated RPV steel. 